\title{
Alcoholism and the Loss of Willpower:
}

\section{A Neurocognitive Perspective}

\author{
Xavier Noël ${ }^{1}$, Antoine Bechara ${ }^{2}$, Damien Brevers ${ }^{1}$, Paul Verbanck ${ }^{1}$, and Salvatore \\ Campanella ${ }^{1}$ \\ ${ }^{1}$ Laboratory of Medical Psychology, CHU-Brugmann, Psychiatry Department, University of \\ Brussels, Belgium \\ ${ }^{2}$ Brain and Creativity Institute, Department of Psychology, University of Southern California, Los \\ Angeles, CA, USA
}

\section{Abstract}

Like other addictions, alcoholism reflects the continuation of alcohol use despite negative consequences (e.g., an ulcer or family problems made worse by alcohol consumption). Recent cognitive theories suggest that optimal information processing related to the capacity to make decisions under uncertainty conditions is impaired either prior to the initiation of alcohol use, or it is related to the consequence of its repeated utilization. In this paper, we suggest that alcoholism may be the product of an imbalance between two separate, but interacting, cognitive registers that contribute to decision making: a reactive/automatic attentional and memory system for signaling the presence of alcohol cues in the environment and for attributing to such cues pleasure and/or excitement; and a reflective/nonautomatic system for regulating the dominant reactive/automatic response. Hyperactivity within the reactive system can override the reflective system and brain/ cognitive changes induced by ethanol could lead to the disruption of self-regulation. We finally develop the idea that different patterns of imbalance between reactive and reflective systems could lead to distinct patterns of clinical impulsivity involved in the vulnerability to, the development of, and the relapse into alcoholism.

\section{Keywords}

alcoholism; decision making; implicit; cognitive biases; inhibition; working memory

\begin{abstract}
Alcoholism is characterized by compulsive preoccupation with obtaining alcohol despite the devastating consequences for one's social and occupational functioning (e.g., in employment, family, education, and health) (American Psychiatric Association, 1994). Like other addictive disorders, individuals with alcoholism are vulnerable to relapse after cessation of drinking (Anton et al., 2006). In this section, we argue that alcoholism is a form of addiction characterized by an imbalance between two separate, but interacting, psychological registers leading to loss of willpower (Bechara, 2005): (1) an impulsive/ reactive register for signaling immediate prospects; (2) a reflective/effortful register needed to moderate the response triggered by the impulsive system. The conditions that lead to this imbalance include (1) a hyperactive reactive system linked notably to an increase in incentive motivational qualities of the drug (e.g., alcohol) and associated stimuli (Robbinson \& Berridge, 1993); and (2) a dysfunctional reflective system including impaired inhibitory
\end{abstract}

(C) 2010 Federation of European Psychophysiology Societies

Xavier Noël: Laboratory of Medical Psychology, Psychiatry Department, Université Libre de Bruxelles, CHU Brugmann, 4, Place Vangehuchten, 1020 Brussels, Belgium, Tel. +32 2 477-2705, Fax +32 2477 2761, xnoel@ulb.ac.be. 
control (Lubman, Yücel, \& Pantelis, 2004). In other words, alcohol can acquire properties of triggering bottom-up, unintentional signals (Stacy, Ames, \& Grenard, 2006) that modulate and bias top-down, goal-driven attentional resources needed for the normal operation of the reflective system and for exercising willpower.

With repeated and reinforced use of alcohol, a variety of internal cues (e.g., stress) and external cues (e.g., a bottle of beer) may come to serve as triggers for well-practiced, affectmodulating patterns of alcohol-use behavior (De Houwer, Crombez, Koster, \& De Beul, 2004; Noël et al., 2005, 2006; Tiffany, 1990; Wiers, van Woerden, Smulders, \& de Jong, 2002). In what follows, we review some possible cognitive candidates that may trigger alcohol use either implicitly (i.e., outside of awareness) or explicitly (i.e., in working memory). Indeed, the study of cognitive biases has become influential in alcohol research (for overviews, see Wiers \& Stacy, 2006). Alcohol-related stimuli (1) capture the attention of problematic users of alcohol (attentional bias); (2) trigger specific attributes (good/bad; sedative/arousal) (memory bias), both of which could increase the likelihood to drink more alcohol or to drink in inappropriate situations (e.g., before an exam or before driving a car). One hypothesis suggests that conscious, explicit expectations influence alcohol use through deliberative decision-making (Goldman, Del Bolca, \& Darkes, 1999); another posits that unconscious memory associations may influence alcohol use more spontaneously (e.g., Wiers \& Stacy, 2006).

Another key characteristic of the problem of drug addiction (including alcoholism) is the reduced capacity for self-control, that is, the efficiency with which individuals can alter their mental representations, impede the execution of certain behaviors and regulate emotions and feelings. In particular, the ability to inhibit or suppress both mental representations recently loaded into working memory and resulting behavior, which is a fundamental aspect of behavioral control, has been the focus of research; chronic use of alcohol produces neural changes leading to general states of "disinhibition" or "dyscontrol" characterized by impulsive and exaggerated behavior, which seems to parallel the acute alcohol effects on behavior (Fillmore, 2003). However, response inhibition is not the only factor involved in the development of alcoholism as well as in relapse; we discuss below the capacity to coordinate dual tasks, to shift from one idea to another, as well as to make advantageous decisions while taking into account not only short-term but also delayed consequences (Finn, 2002; Noël, Van der Linden, \& Verbanck, 2008). The latter point is illustrated by Damasio (1994, p. 175): "Willpower is just another name for the idea of choosing according to long-term outcomes rather than short-term ones." According to this theorist, willpower depends in many important ways on neural substrates that regulate homeostasis, emotion, and feeling. Recent advances in the comprehension of decision-making under ambiguity in healthy participants (Persaud, McLeod, \& Cowey, 2007), in neurological patients (Bechara, Damasio, Tranel, \& Damasio, 1997), and in addicted people (Bechara, 2005) strongly suggest a case for a somatic marker model of alcoholism.

Together, these recent advances in our understanding of alcoholism constitute promising new ways in which abnormal cognitive and affective responses triggered by certain stimuli in relation to alcohol use could be altered to weaken their strength and therefore improve the exercise of will.

\section{Alcohol Cognitive Biases}

During the past decade, research on cognitive biases has become influential in the study of the problematic use of alcohol and alcoholism (for a overview, see Wiers \& Stacy, 2006). Cognitive bias approaches can be roughly divided into three lines of research with their own historical roots and background in science. The first is the attentional bias approach, which 
demonstrates that alcohol-related information captures the attention of heavy drinkers and alcoholics (e.g., Johnsen, Laberg, Cox, Vaksdal, \& Hugdahl, 1994; Noël et al., 2006). In other words, the practice of an abnormal level of drinking appears to be very closely linked to attentional biases toward alcohol cues.

Another main avenue of research has focused on memory biases, which are viewed as strong candidates for alcohol use (Stacy, Ames, \& Grenard, 2006). Thus, the second line of research investigated the influence of explicit cognitions/expectations (e.g., social facilitation, relaxation) concerning the effect of alcohol on drinking behavior (e.g., Goldman et al., 1999). This approach suggests that conscious, explicit expectations influence alcohol use through deliberative decision making. Third, memory activation processes could be investigated with implicit memory tasks to identify implicit cognitions, which could be defined as "introspectively unidentified (or inaccurately identified) traces of past experience that mediate feeling, thought, or action" (Greenwald \& Banaji, 1995). According to this approach, unconscious memory associations may influence alcohol use. For instance, an implicit association task showed that the strength of the association between alcohol and arousal was stronger in heavy drinkers than in recreational drinkers of alcohol (Wiers et al., 2002). Importantly, the use of implicit as well as explicit measures may be complementary because they tap different underlying cognitive-motivational processes.

\section{Attentional Biases for Alcohol Cues}

Repeated drug/alcohol use is associated with a bias toward attending to drug-related rather than to other stimuli (e.g., Johnsen et al., 1994; Noël et al., 2006). For instance, on a modified version of the Stroop task (often inappropriately called "emotional Stroop task"), alcoholics named the color of alcohol-related words much slower than matched neutral words, a difference that was absent in control, nonalcoholic participants.

In a task consisting of identifying transient changes in visual scenes, people drinking "heavily" detected substance-related changes more quickly than light and nonalcohol drinkers (Jones, Jones, Smith, \& Copley, 2003). This is consistent with the idea that alcohol cues "hijack" limited attentional resources and thereby reduce the processing resources available for other cognitive functions. Also, when presented with alcohol-related words on the periphery of their visual field, alcoholics were poorer at completing a centrally presented odd/even number decision task than when either nonalcohol related words, nonwords, or no peripherally presented stimuli were present (Waters \& Green, 2003). Taken together, these findings demonstrate that alcohol cues command limited attentional resources and thereby reduce the processing resources available for other cognitive functions.

The involvement of attentional biases in alcohol consumption after a period of abstinence was demonstrated by Cox and colleagues (Cox, Hogan, Kristian, \& Race, 2002), who found attentional bias for alcohol cues to be a reliable predictor of alcohol relapse during a detoxification treatment. Furthermore, the intensity of self-reported craving for alcohol was correlated to their proneness to maintain their attention toward alcohol-related pictures (Field, Mogg, Zetteler, \& Bradley, 2004). In sum, these data suggest that attentional bias for alcohol cues is involved in alcohol relapse.

An additional important question concerns the time-course of attention for alcohol cues and its relationship with the motivation to drink alcohol (e.g., Field et al., 2004; Loeber et al., 2009; Noël et al., 2006). In an alcohol visual dot probe task, immediately after the offset of the picture pair (one related to alcohol, e.g., man holding beer glass to mouth, bottles of whisky, and one not related to alcohol), a dot probe was presented in the position of one of the two preceding pictures. Participants were instructed to press, as quickly as possible, one of the two response buttons to indicate the location of the probe. Relative to social drinkers 
of alcohol, recently detoxified alcoholics had an enhanced attentional bias for alcoholrelated pictures presented at the shortest stimulus duration $(50 \mathrm{~ms})$, thus indicating the presence of initial-automatic orienting attentional bias (Loeber et al., 2009; Noël et al., 2006). When pictures were presented for $500 \mathrm{~ms}$, these patients were not quicker in detecting a probe replacing alcohol-related pictures, thus attesting the absence of attentional bias (Noël et al., 2006). In contrast, recreational drinkers of alcohol exhibited the inverse attentional pattern, that is, no attentional preference for alcohol-related pictures presented for $50 \mathrm{~ms}$ and an enhanced attentional bias for alcohol-related pictures displayed for $500 \mathrm{~ms}$. In addition, the severity of alcoholism measured by the total number of prior detoxification treatments was positively correlated with the bias of attentional attraction for alcohol pictures presented for $50 \mathrm{~ms}$. The observed pattern in alcoholics suggest that, following treatment, alcohol-dependent individuals show a bias to avoid alcohol-related cues, after initially orienting their attention to those cues, i.e., an "approach-avoidance" pattern of attention bias.

Franken (2003) reviewed both the psychopharmacological and neuroanatomical perspectives on the neural basis of attentional biases and suggested that when conditioned drug stimuli are present, they increase dopamine levels in the corticostriatal circuit, particularly in the anterior cingulate cortex, amygdala, and nucleus accumbens, which in turn serves to draw the subject's attention toward the drug-related stimulus. In line with this hypothesis, the incentive sensitization theory (Robinson \& Berridge, 1993) posits that addictive drugs enhance mesolimbic dopamine transmission and thereby constitute the neural substrate of the attribution of incentive salience to the perception and mental representation of events associated with the activation of this system. In psychological terms, when environmental stimuli are associated with drug use, they acquire conditioned incentive properties, so that drug-related stimuli become perceived as highly attractive, thereby "grabbing" attention.

\section{Explicit and Implicit Alcohol-Related Associations}

Explicit cognitions are the beliefs individuals hold about the effects of alcohol on behavior, moods, and emotions. Expectancies about the effects of alcohol (e.g., expected relaxation or social facilitation effects) predict up to 50\% of the variance in alcohol use (Goldman et al., 1999). Also, expectancies measured in young adolescents significantly predict drinking patterns up to 9 years later, after controlling for earlier alcohol and drug use (Stacy, Newcomb, \& Bentler, 1991).

However, there are several limitations to such a direct and explicit approach. First, explicit cognitions rely on self-report, which means that the only information that can be collected is that available from introspection. Second, this approach is very sensitive to self-justification and social desirability. For these reasons, Greenwald and Banaji (1995) called for the use of more indirect assessment of attitudes and related concepts in addition to explicit assessment. As a definition, implicit cognitions could be defined as "introspectively unidentified (or inaccurately identified) traces of past experience that mediate feeling, thought, or action" (Greenwald \& Banaji, 1995). In addition, the use of implicit as well as explicit measures may be important because they may tap different underlying cognitive-motivational processes (Stacy et al., 1991).

The most well-known way to assess these cognitions is the Implicit Association Test (IAT; Greenwald, McGhee, \& Schwarts, 1998). In a typical IAT, stimuli belonging to one of four possible categories are presented one by one on a computer screen. On each trial, participants categorize the presented stimulus by pressing one of two keys. The assumption underlying the IAT is that performance should be better in a task where associated categories are assigned to different responses. For instance, when classifying names of flowers or insects, positive or negative words, people are faster when flowers and positive 
words are assigned to one key and insects and negative words to the second key than they are when the first key in assigned to insects and positive words and the second key to flowers and negative words (Greenwald et al., 1998). Therefore, if performance depends on which categories are assigned to the same response, this provides information about the relative strength of the associations between the concepts (De Houwer, 2002). Applied to alcohol use and misuse, such research examined whether light and heavy drinkers differ in their implicit attitude toward alcohol, that is to say, the extent to which they implicitly associate alcohol with positive and negative valence (De Houwer et al., 2004; Wiers et al., 2002). To do this, positive and negative words, the names of alcoholic beverages and of sodas had to be categorized by light and heavy drinkers of alcohol. In a second IAT, positive and negative words were replaced by active (e.g., excited) or passive words (e.g., relaxed). Interestingly, the results indicated that both light and heavy drinkers (higher weekly alcohol use and more alcohol-related problems) exhibited a more negative implicit attitude toward alcohol drinks than toward sodas - but only heavy drinkers were faster at categorizing alcohol-related words with active words (Wiers et al., 2002). Similar results were found in detoxified abstinent alcoholics at an alcohol-rehabilitation clinic (De Houwer et al., 2004). These findings are predicted by the incentive-motivation theory of Robinson and Berridge (Robinson \& Berridge, 1993), according to which the transition between the use of a psychoactive substance and its misuse may be caused by sensitized arousal for drug cues (leading to enhanced "wanting") and this independently to "liking" attitudes. Moreover, arousal associations may act in an unconscious information-processing level (Robinson \& Berridge, 1993; Wiers et al., 2002).

Most studies assessing implicit cognitive processes in addictive behaviors have used drugrelated stimuli that were not personality selected for each participant. One main limitation is that addiction-related stimuli cannot apply to all participants, who vary considerably from each other in terms of their addiction-related habits and preferences. For instance, in the case of alcohol consumption, participants might know little about alcoholic beverages or brand names that are not used regularly, which may lead to an underestimation of the size of attentional and memory biases. Even more, an alcoholic for whom beers are the favorite beverage might find wine, for instance, disgusting. For this reason, it might be recommended to use personalized stimuli to measure implicit association.

\section{Alcoholism and Impaired Effortful Processes}

Numerous studies have shown that chronic alcohol consumption is associated with severe executive function deficits, which are still present after a protracted period of alcohol abstinence (e.g., Noël et al., 2001; Oscar-Berman, Kirkley, Gansler, \& Couture, 2004). By definition, executive functioning encompasses a series of high-level processes, the main aim of which is to facilitate adaptation to new or complex situations, when highly practiced cognitive abilities no longer suffice (Shallice, 1994). Research showed that recently detoxified alcoholics are generally less efficient than their controls when required to carry out two tasks simultaneously (Noël et al., 2001; Rapeli, Service, Salin, \& Holopainen, 1997), to inhibit a prepotent response (Dao-Castellana et al., 1998), to detect rules (Noël et al., 2001), to shift between multiple sets of responses (Pitel et al., 2007), to plan (Joyce \& Robbins, 1991), and to generate concepts (Knight \& Longmore, 1996). In addition, in vivo structural neuroimaging studies confirmed the presence in frontal lobes of substantial volume shrinkage, proton metabolite abnormality, glucose metabolic and perfusion impairment in sober alcoholics (Sullivan \& Pfefferbaum, 2005). Postmortem analyses showed neuronal loss restricted to the superior frontal cortex (Kril \& Halliday, 1997). Functional imagery such as fMRI, which enables the detection of blood flow response to the activation of specific brain regions by motor, sensory, or cognitive processes showed abnormal patterns of activation in alcohol abusers during cognitive tasks assessing their 
capacity to manipulate information stored in working memory (Schweinsburg et al., 2005) and assessing inhibitory control (Dao-Castellana et al., 1998; Li, Luo, Yan, Bergquist, \& Sinha, 2009). Taken together, behavioral and brain imaging data support the idea that the cognitive deficits in recently detoxified sober alcoholics are due, at least partly, to a frontal lobe dysfunction (for an overview, see Moselhy, Georgiou, \& Kahn, 2001).

Several studies (e.g., Noël et al., 2001) used executive tasks coherently designed according to Norman and Shallice's control of action model (Shallice, 1994) and Baddeley's working memory model (Baddeley, 1986). Briefly, Shallice's model distinguishes two control to action mechanisms. The first, contention scheduling, is involved in routine situations in which actions are triggered automatically. The second, the supervisory attentional system (SAS), is needed in situations where the routine selection of action is unsatisfactory, and is conceived as carrying out a variety of processes allowing the genesis of plans and willed actions.

An analogy has been established between the SAS and the central executive component of the working memory model proposed by Baddeley (Baddeley, 1986). Working memory is a limited capacity system responsible for the temporary storage and processing of information while cognitive tasks are performed. Baddeley's model comprises two slave systems ensuring temporary maintenance of information, the phonological loop and the visuospatial sketchpad, and an attentional control system, the central executive, the function of which is similar to that of the SAS. According to this model, an important function of the central executive is to allow two tasks to be carried out simultaneously.

An alpha-span task can be used in accordance with this model to investigate the ability to manipulate information stored in working memory by comparing the recall of information in serial order (involving mainly a storage component) and in alphabetical order (involving storage and manipulation of information) (Belleville, Rouleau, \& Caza, 1998). The results of such a task in alcoholics show that their normal storage component (measured by the span size and the score of the serial condition) remains healthy, whereas their ability to manipulate the information stored (measured by the alphabetic condition) is impaired (Noël et al., 2001).

Prepotent response inhibition - a main function of the supervisory attentional system - was assessed with the Hayling task (Burgess \& Shallice, 1996), which consists of two sections (A and B) of 15 sentences each, in which the last word was missing and strongly primed by the meaning of the sentence (e.g., "Most cats see very well at ..."). Sentences were read aloud by the experimenter. In section A (initiation/automatic) subjects were asked to give the word that made sense (e.g., "Most cats see very well at night"). In section B (inhibition), participants were asked to give a word that made no sense at all in the context of the sentence (e.g., "Most cats see very well at ... 'elephant"'). On this inhibition test, recently detoxified men with alcoholism were slower on the inhibition section, but not on the section requiring them to produce an automatic response. They also made more inhibition errors than their controls (Noël et al., 2001, 2002).

Motor response inhibition of problematic users of alcohol has been repeatedly examined with different versions of the Go/No-Go paradigm (for a review, see Kamarajan et al., 2005). This task generally involves participants making a response to a target (pressing a key; Go response) while withholding their response to a distractor (No-Go response). Behavioral analyses showed that alcoholics on average made 30-40\% more No-Go errors than matched control participants (Noël, Van der Linden et al., 2007). Two of the electrophysiological signatures that characterize these behaviors have also been reported in the literature. First, the "No-Go N2," a negative deflection with a frontocentral maximum 
around 200-300 ms, and second, the "No-Go P3," an augmented positive-going peak usually between 300 and $600 \mathrm{~ms}$ (Kamarajan et al., 2005). A number of studies provide strong evidence that the fronto-central maximum No-Go hypotensions (No-Go P300) were suppressed in alcoholic subjects compared to controls (Cohen, Porjesz, Begleiter, \& Wang, 1997). In addition, No-Go responses associated with theta oscillations in alcoholics were found to be significantly attenuated in the frontal region (Kamarajan et al., 2004).

In separate studies (Noël et al., 2005; Noël, Van der Linden et al., 2007), we tested the hypotheses that alcoholics suffer from deficits in their cognitive control mechanisms of "stopping" and of "shifting," and that these deficits are exacerbated by cognitive biases for alcohol-related stimuli. The Alcohol Shifting Task involves the presentation of alcoholrelated versus nonalcohol-related words on a computer screen. Sometimes, the alcoholrelated words were the targets for the "Go" response, with neutral words as distracters, sometimes the reverse. Several shifts in the type of the target occurred during the task. Relative to control participants, we found that alcoholics were slower to respond to neutral, but not to alcohol stimuli in both stopping and shifting conditions. In addition, when subjects were supposed to withhold their response to an alcohol or neutral stimulus, alcoholics made more errors than controls in both "stopping" and "shifting" conditions. Most important, when alcohol-related stimuli had to be detected, alcoholics showed a greater number of correct Go responses and also more signs of disinhibition, indicating an inhibition bias associated with alcoholism.

The clinical impact of the working memory's central executive could be important. Indeed, dual-task capacity and inhibitory control deficits observed in alcohol-detoxified subjects could be critical factors responsible for the alcoholic's weak capacity to remain abstinent within a 3-month period after detoxification (e.g., Noël et al., 2002).

A very challenging question still remains: Could deficits of inhibition have been a cause of alcoholics becoming addicted rather than a consequence of their addiction? In support of the hypothesis that at least some inhibition/shifting deficits may predate the onset of alcohol dependence, in Go/No-Go tasks the anteriorly distributed No-Go P3 potential has a markedly reduced amplitude in high-risk individuals (e.g., children of alcoholics), indicating impaired inhibitory control in these individuals (Pfefferbaum, Ford, White, \& Mathalon, 1991). More recently, an fMRI study compared youths with and without a family history of alcoholism, using a Go/No-Go procedure, and demonstrated that youths with a family history of alcoholism showed less inhibitory frontal response than other youths, suggesting that inhibition deficits could be indeed be predisposing factors to alcohol abuse (Schweinsburg et al., 2004).

\section{Between Automatisms and Effortful Processes: The Role of Insula in Conscious Alcohol Urge}

Evidence points toward a crucial role of the insula in conscious drug urges and in translating interoceptive signals into conscious feelings and behavioral biases during decision making that involved uncertain risk and reward (Naqvi \& Bechara, 2009). Over time, as addiction increases, stimuli within the environment associated with drug use become powerful incentives, initiating both automatic (implicit) motivational processes that drive ongoing drug use and relapse as well as conscious (explicit) feelings of urges to take drugs. Once the insula is engaged, it does two things: (1) It potentiates or enhances the activity of the habitual-automatic-IAT system; (2) it weakens or further disables the capacity of the inhibitory control system. The crucial role of the insula in addiction was recently revealed in tobacco smokers (Naqvi \& Bechara, 2009). In their study, Naqvi and Bechara showed that, when comparing 19 smokers who had sustained damage in the insula with another 50 
smokers who had sustained damage in other brain areas, the former were 100 times more likely than the latter to undergo a "disruption of smoking addiction," characterized by the ability to quit easily, immediately, without relapse and without the persistence of the urge to smoke.

Another way in which the interoceptive effects of alcohol use might contribute to the urge to take drugs is during drug withdrawal. Long-term use of most drugs of abuse results in adaptations in multiple neural, endocrine, and visceral systems expressed when drug use is discontinued. With alcohol, for example, cessation of long-term use results in tachycardia, hypertension, sweating, tremor, and, if left untreated, delirium, seizures, and even death. The physiological state of withdrawal is usually short-lived, lasting days to weeks. Subjectively, withdrawal is associated with dysphoria, along with an intense urge to take the drug. Withdrawal urges might be seen as a subjective need to alleviate the dysphoria (Koob \& Bloom, 1988). The insula might function to translate the physiological state of withdrawal, with all of its interoceptive components, into subjective dysphoria toward the appropriate goal object (i.e., the nucleus accumbens and associated mesolimbic dopamine system). On the other hand, the insula activation might impact the prefrontal cortex functions so that it can subvert attention, reasoning, planning, and decision-making processes to formulate plans for action to seek and procure drugs (Contreras, Ceric, \& Torrealba, 2007). Put differently, these interoceptive representations have the capacity to "hijack" the cognitive resources necessary for exerting inhibitory control to resist drug use (Bechara, 2005).

\section{Alcoholism and Decision-Making Under Ambiguity and Under Risk}

In everyday life, decision-making situations differ in their degree of probability or associated reward and punishment. Decisions "under ambiguity" characterize those situations for which the outcome of an individual's choices is both uncertain and not defined by clear probabilities. In contrast, when situations provide a known distribution of possible outcomes, this aspect of uncertainty is best conceptualized as "decision making under risk" (Platt \& Huettel, 2008).

For several years, research has considered alcoholism to be paradigmatic of impaired decision-making under ambiguity (Bechara, 2005). Indeed, as a typical cognitive task assessing decision making under ambiguity, the Iowa Gambling Task (IGT, Bechara, Damasio, Damasio, \& Anderson, 1994) requires individuals to choose between several alternatives. The choices (selection of cards from different decks) are either advantageous or disadvantageous, but each choice is ambiguous - at least initially -with regard to the outcome. Using this task, it was shown that, compared to normal controls, a high proportion of participants detoxified and abstinent for a few weeks (Goudriaan, Oosterlaan, de Beurs, \& van den Brink, 2005) or even for several years (Fein, Klein, \& Finn, 2004) performed poorly on the IGT, i.e., they make choices that bring immediate reward, but then lead to more severe delayed punishment. Furthermore, some studies showed that performance on the IGT was best predicted by an index derived from several measures, including the severity of one's addiction (e.g., quantity of drug used, number of detoxification treatments), and especially the ability to hold gainful employment, thus providing ecological validity of the IGT in assessing decision making in addicted individuals (Bechara et al., 2001).

The main problem with this task is that identification of the more specific cognitive processes that may be responsible for poor performance remains unclear (for a review, see Dunn, Dalgleish, \& Lawrence, 2006). For instance, some studies have suggested as in patients with lesions in the ventromedian prefrontal cortex (VMPC) (Damasio, 1994), poor performance of the IGT in alcoholics and addicts to illicit drugs is due to impaired activation of somatic markers (Bechara, Dolan, \& Hindes, 2002). 
The somatic marker hypothesis is a systems-level neuroanatomical and cognitive framework for choosing according to long-term, rather than short-term, outcomes (Damasio, 1994). The key idea of this hypothesis is that the process of decision making depends in many important means on neural substrates that regulate homeostasis, emotion, and feeling. The term "somatic" refers to the collection of body- and brain-related responses that hallmark affective and emotional responses. Both the amygdala and the VMPC are critical for triggering somatic states; but the amygdala responds to events that occur in the environment, whereas the VMPC triggers somatic states from memories, knowledge, and cognition (Bechara, 2005). More specifically, during the process of pondering decisions, the immediate and future prospects of an option may trigger numerous affective (somatic) responses that conflict with each other; the end result is that an overall positive or negative signal emerges. While normal individuals experience a state of arousal during the time of deliberation prior to making disadvantageous choices, the insensitivity to future consequences ("myopia for the future") seen in VMPC patients is thought to be derived from their failure to experience this affective state. This affective experience is normally accompanied by bodily signals and somatic states that have become associated with risky decisions; these bodily signals (or somatic markers) are derived from prior experiences with reward and punishment. This mechanism of deficient activation of somatic markers has been suggested as explaining poor IGT performance in a subgroup of alcohol abusers (Bechara et al., 2002). Another mechanism that might possibly underlie poor IGT performance in alcohol abusers could be hypersensitivity to reward (Bechara et al., 2002). Indeed, poor decision making shown by a subgroup of substance-dependent individuals with alcoholism has been attributed to exaggerated reactions to reward.

A further aspect of this theory is that these somatic markers can be unconscious: They can bias the response selection even when a person is unaware of them (Damasio, 1994; Persaud et al., 2007). A closer inspection of processes subserving decision making in the IGT also reveals the critical importance of effortful processes related to response inhibition, mental shifting, memory, including the maintenance of active representations of certain information for a short period (i.e., several 10s of seconds) in working memory, or for a longer period of time in episodic memory (Dunn et al., 2006).

\section{Conclusions: Directions for Future Research and Implication for Treatment}

Advances in the study of cognition, emotion, and their interactions have improved our understanding of the implicit and explicit cognitive mechanisms that govern the misuse of and dependence on alcohol. Cognitive deficits in individuals with alcoholism are mainly intentional/executive controls per se (e.g., response inhibition, shifting) and their deleterious impact on memory systems such as working and episodic memory. Cognitive biases are related to the way in which attention is captured and maintained by alcohol-related information, and also implicit and memory biases in semantic, episodic and procedural memory. The contribution of these cognitive characteristics to the vulnerability to alcoholism and to relapse once detoxified is now well supported by research data.

We argued that, in alcoholics, the interaction between cognitive biases and impaired executive functions together with abnormal affective processing could result in weakened willpower, that is, when long-term consequences of an action modulate decision making. In other words, the inability to make choices according to long-term outcomes can result from a dysfunction of one or more of several mechanisms of cognitive and behavioral control within the reflective system, or hyperactivity of the reactive system.

The relationship remains to be clarified between, on the one hand, emotions (positive vs. negative), attentional and memory biases for alcohol cues, executive functioning, and 
decision making and, on the other hand, the development of dependence on alcohol and the tendency to relapse. For instance, attentional biases toward alcohol-associated pictures were underlined in alcoholic patients dependent for less than 9 years but not in patients with a longer duration of dependence (Loeber et al., 2009) and in patients with impaired attention and working memory deficits, thus suggesting a change of underlying processes from early to late stages of alcohol dependence.

Finally, somatic markers impaired during decisions made under ambiguity may enhance the risk for individuals to become problematic users of alcohol because they select optional decisions with positive outcomes and/or expectations in the short term (arousal, social facilitation, etc.) to the detriment of their adaptation to consequences in the long run. Numerous impaired automatic (emotional reactions to an aversive stimulus) and effortful (intentional response inhibition, shifting) processes could explain poor choice adjustment to delay consequences. Episodic memory disorders and cognitive capacities associated, for instance, with time travel, are also good candidates to explain impaired decision making in alcoholic subjects and certainly deserve further investigation.

Future research should address the reasons why these mechanisms break down: Are genetic predispositions necessary to becoming an addict via abnormal neurotransmitter transporters? Are the mechanisms developmental, for example, the result of environmental stress or exposure to alcohol during a time window in childhood or in adolescence when brain maturation has not yet developed completely? Are there multiple developmental pathways to becoming a true addict to alcohol, which may explain the heterogeneity of alcoholics in term of age of onset, of personality disorders, of the use of illicit drugs, and thus also of the efficacy of psychological treatments and medication?

A better characterization of the cognitive and affective mechanisms that increase an individual's vulnerability to alcohol and to relapse could also lead to more efficient psychological treatments and medication (Mann, Kiefer, Spanagel, \& Littleton, 2008). On the basis of the dual-process theory developed in this article, an intervention could be aimed at more explicit and/or more implicit cognitive processes. For instance, diminishing attentional biases with the help of specific cognitive procedures (Wiers \& Stacy, 2006) and revalidating inhibitory and executive control could lead to decreased risk of experiencing alcohol misuse and relapse.

\section{References}

American Psychiatric Association. Diagnostic and statistical manual of mental disorders. 4. Washington, DC: Author; 1994.

Anton R, O’Malley S, Ciraulo D, Cisler R, Couper D, Donovan D, Zweben A. COMBINE study research group. Combined pharmacotherapies and behavioral interventions for alcohol dependence: the COMBINE study: A randomized controlled trial. Journal of the American Medical Association. 2006; 295:2003-2017. [PubMed: 16670409]

Baddeley, A. Working memory. Oxford: Clarendon; 1986.

Bechara A. Decision making, impulse control and loss of willpower to resist drugs: A neurocognitive perspective. Nature Neuroscience. 2005; 8:1458-1463.

Bechara A, Damasio A, Damasio H, Anderson S. Insensitivity to future consequences following damage to human prefrontal cortex. Cognition. 1994; 50:7-15. [PubMed: 8039375]

Bechara A, Damasio H, Tranel D, Damasio A. Deciding advantageously before knowing the advantageous strategy. Science. 1997; 28:1293-1295. [PubMed: 9036851]

Bechara A, Dolan S, Denburg N, Hindes A, Anderson S, Nathan P. Decision-making deficits, linked to a dysfunctional ventromedial prefrontal cortex, revealed in alcohol and stimulant abusers. Neuropsychologia. 2001; 39:376-389. [PubMed: 11164876] 
Bechara A, Dolan S, Hindes A. Decision-making and addiction. Part II: Myopia for the future or hypersensitivity to reward? Neuropsychologia. 2002; 40:1690-1705. [PubMed: 11992657]

Belleville S, Rouleau N, Caza N. Effects of normal aging on the manipulation of information in working memory. Memory and Cognition. 1998; 26:572-583.

Burgess P, Shallice T. Response suppression, initiation and strategy use following frontal lobe lesions. Neuropsychologia. 1996; 34:263-273. [PubMed: 8657357]

Cohen H, Porjesz B, Begleiter H, Wang W. Neuroelectric correlated of response production and inhibition in individuals at risk to develop alcoholism. Biological Psychiatry. 1997; 42:57-67. [PubMed: 9193742]

Contreras M, Ceric F, Torrealba F. Inactivation of the interoceptive insula disrupts drug craving and malaise induced by lithium. Science. 2007; 318:655-658. [PubMed: 17962567]

Cox W, Hogan L, Kristian M, Race J. Alcohol attentional bias as a predictor of alcohol abusers' treatment outcome. Drug and Alcohol Dependence. 2002; 68:237-243. [PubMed: 12393218]

Damasio, A. Descartes' error: Emotion: Reason and the human brain. New York: Grosset/Putnam; 1994.

Dao-Castellana M, Samson Y, Legault F, Martinot J, Aubin H, Crouzel C, Synota A. Frontal dysfunction in neurologically normal chronic alcoholic subjects: Metabolic and neuropsychological findings. Psychological Medicine. 1998; 28:1039-1048. [PubMed: 9794011]

De Houwer J. The implicit association test as a tool for studying dysfunctional associations in psychopathology: Strengths and limitations. Journal of Behavior Therapy and Experimental Psychiatry. 2002; 33:115-133. [PubMed: 12472175]

De Houwer J, Crombez G, Koster E, De Beul N. Implicit alcohol-related cognitions in a clinical sample of heavy drinkers. Journal of Behavior Therapy and Experimental Psychiatry. 2004; 35:275-286. [PubMed: 15530843]

Dunn B, Dalgleish T, Lawrence A. The somatic marker hypothesis: A critical evaluation. Neuroscience Biobehavioral Reviews. 2006; 30:239-271.

Fein G, Klein L, Finn P. Impairment on a simulated gambling task in long-term abstinent alcoholics. Alcoholism: Clinical and Experimental Research. 2004; 28:1487-1491.

Field M, Mogg K, Zetteler J, Bradley B. Attention biases for alcohol cues in heavy and light social drinkers: The roles of initial orienting and maintained attention. Psychopharmacology. 2004; 176:105-111.

Fillmore M. Drug abuse as a problem of impaired control: Current approaches and findings. Behavioral and Cognitive Neuroscience Reviews. 2003; 2:179-197. [PubMed: 15006292]

Finn P. Motivation, working memory, and decision making: A cognitive-motivational theory of personality to alcoholism. Behavioral and Cognitive Neuroscience Reviews. 2002; 1:183-205. [PubMed: 17715592]

Franken I. Drug craving and addiction: Integration psychological and neuropsychopharmacological approaches. Progress in Neuro-Psychopharmacology and Biological Psychiatry. 2003; 27:88-93.

Goldman, M.; Del Boca, F.; Darkes, J. Alcohol expectancy theory: The application of cognitive neuroscience. In: Blane, HT.; Leonard, K., editors. Psychological theories of drinking and alcoholism. New York: Guilford; 1999. p. 203-246.

Goudriaan A, Oosterlaan J, de Beurs E, van den Brink W. Decision making in pathological gambling: A comparison between pathological gamblers, alcohol dependents, persons with Tourette syndrome, and normal controls. Cognitive Brain Research. 2005; 23:137-151. [PubMed: 15795140]

Greenwald A, Banaji M. Implicit social cognition: Attitudes, self-esteem, and stereotypes. Psychological Review. 1995; 102(1):4-27. [PubMed: 7878162]

Greenwald A, McGhee D, Schwarts J. Measuring individual differences in implicit cognition: The Implicit Association Test. Journal of Personality and Social Psychology. 1998; 74:1464-1480. [PubMed: 9654756]

Johnsen B, Laberg J, Cox W, Vaksdal A, Hugdahl K. Alcoholic subjects' attentional bias in the processing of alcohol-related words. Psychology of Addictive Behavior. 1994; 8:111-115. 
Jones B, Jones B, Smith H, Copley N. A flicker paradigm for inducing change blindness reveals alcohol and cannabis information processing biases in social users. Addiction. 2003; 98:235-244. [PubMed: 12534429]

Joyce EM, Robbins TW. Frontal lobe function in Korsakoff and non-Korsakoff alcoholics: Planning and spatial working memory. Neuropsychologia. 1991; 29:709-723. [PubMed: 1944873]

Kamarajan C, Porjesz B, Jones K, Choi K, Chorlian D, Padmanabhapillai A, Begleiter H. The role of brain oscillations as functional correlates of cognitive systems: A study of frontal inhibitory control in alcoholism. International Journal of Psychophysiology. 2004; 51:155-180. [PubMed: 14693365]

Kamarajan C, Porjesz B, Jones K, Choi K, Chorlian D, Padmanabhapillai A, Begleiter H. Alcoholism is a disinhibitory disorder: Neurophysiological evidence from a Go/No-Go task. Biological Psychology. 2005; 69:353-373. [PubMed: 15925035]

Knight, R.; Longmore, B. Clinical neuropsychology of alcoholism. Hove, UK: Erlbaum; 1994.

Koob GF, Bloom FE. Cellular and molecular mechanisms of drug dependence. Science. 1988; 242:715-723. [PubMed: 2903550]

Kril J, Halliday G. The cerebral Cortex is damaged in chronic alcoholics. Neuroscience. 1997; 79:983998. [PubMed: 9219961]

Li CS, Luo X, Yan P, Bergquist K, Sinha R. Altered impulse control in alcohol dependence: Neural measures of stop signal performance. Alcoholism: Clinical and Experimental Research. 2009; 33:740-750.

Loeber S, Vollstädt-Kein S, von der Goltz C, Flor H, Mann K, Keifer F. Attentional bias in alcoholdependent patients the role of chronicity and executive functioning. Addiction Biology. 2009; 14:194-203. [PubMed: 19291010]

Lubman D, Yücel M, Pantelis C. Addiction, a condition of compulsive behavior? Neuroimaging and neuropsychological evidence of inhibitory dysregulation. Addiction. 2004; 99:1491-1502. [PubMed: 15585037]

Mann K, Kiefer F, Spanagel R, Littleton J. Acamprosate: Resent findings and future research directions. Alcoholism: Clinical and Experimental Research. 2008; 32:1105-1110.

Moselhy H, Georgiou G, Kahn A. Frontal lobe changes in alcoholism: A review of the literature. Alcohol and Alcoholism. 2001; 36:357-368. [PubMed: 11524299]

Naqvi NH, Bechara A. The hidden island of addiction: The insula. Trends in Neurosciences. 2009; 32(1):56-67. [PubMed: 18986715]

Noël X, Colmant M, Van der Linden M, Bechara A, Bullens Q, Hanak C, Verbanck P. Time course of attention for alcohol cues in abstinent alcoholic patients: The role of initial orienting. Alcoholism: Clinical and Experimental Research. 2006; 30:1871-1877.

Noël X, Sferrazza R, Van der Linden M, Paternot J, Verhas M, Hanak C, Verbanck P. Median frontal hypo-metabolism measured by $99 \mathrm{mTc}$-Bicisate SPECT procedure in the prediction of short term alcohol abstinence in alcohol-dependent patients. Alcohol and Alcoholism. 2002; 37:347-354. [PubMed: 12107037]

Noël X, Van der Linden M, d'Acremont M, Bechara A, Dan B, Hanak C, Verbanck P. Alcohol cues increase cognitive impulsivity in individuals with alcoholism. Psychopharmacology. 2007; 192:291-298. [PubMed: 17279375]

Noël X, Van der Linden M, d'Acremont M, Colmant M, Hanak C, Pelc I, Bechara A. Cognitive biases toward alcohol-related words and executive deficits in polysubstance abusers with alcoholism. Addiction. 2005; 100:1302-1309. [PubMed: 16128719]

Noël X, Van der Linden M, Schmidt N, Sferrazza R, Hanak C, Le Bon O, Verbanck P. Supervisory attentional system in nonamnesic male alcoholic subjects. Archives of General Psychiatry. 2001; 58:1152-1158. [PubMed: 11735844]

Noël, X.; Van der Linden, M.; Verbanck, P. Abus de substances psychoactives [Psychoactive substance abuse]. In: Van der Linden, M.; Ceschi, G., editors. Traité de Psychopathologie Cognitive: Etats Psychopathologiques, Tome II. Marseille: Solal; 2008. p. 225-248.

Oscar-Berman M, Kirkley SM, Gansler DA, Couture A. Comparisons of Korsakoff and non-Korsakoff alcoholics on neuropsychological tests of prefrontal brain functioning. Alcoholism: Clinical and Experimental Research. 2004; 28:667-675. 
Persaud N, McLeod P, Cowey A. Postdecision wagering objectively measures awareness. Nature Neuroscience. 2007; 10:257-261.

Pfefferbaum A, Ford J, White P, Mathalon D. Event-related potentials in alcoholic men: P3 amplitude reflects family history but not alcohol consumption. Alcoholism: Clinical and Experimental Research. 1991; 15:839-850.

Pitel AL, Beaunieux H, Witkowski T, Vabret F, Guillery-Girard B, Quinette P, Eustache F. Genuine episodic memory déficits and executive dysfunctions in alcoholic subjects early in abstinence. Alcoholism: Clinical and Experimental Research. 2007; 31:1169-1178.

Platt M, Huettel S. Risky business: The neuroeconomics of decision making under uncertainty. Nature Neuroscience. 2008; 11:398-403.

Rapeli P, Service E, Salin P, Holopainen A. A dissociation between simple and complex span impairment in alcoholics. Memory. 1997; 5:741-762. [PubMed: 9497910]

Robinson T, Berridge K. The neural basis of drug craving: An incentive-sensitization theory of addiction. Brain Research Reviews. 1993; 18:247-291. [PubMed: 8401595]

Robinson T, Berridge K. Addiction. Annual Review of Psychology. 2003; 54:25-53.

Schweinsburg A, Paulus M, Barlett V, Killeen L, Caldwell L, Pulido C, Tapent SF. An FMRI study of response inhibition in youths with a family history of alcoholism. Annals of the New-York Academy of Sciences. 2004; 1021:391-394. [PubMed: 15251915]

Schweinsburg A, Schweinsburg BC, Cheung EH, Brown GG, Brown SA, Tapert SF. fMRI response to spatial working memory in adolescents with comorbid marijuana and alcohol use disorders. Drug and Alcohol Dependence. 2005; 79:201-210. [PubMed: 16002029]

Shallice, T. Multiple levels of control processes. In: Umita, C.; Moscovitch, M., editors. Attention and performance, XV. Conscious and nonconscious information. Cambridge: A Bradford Book/The MIT; 1994. p. 395-420.

Stacy, A.; Ames, SL.; Grenard, JL. Word association tests of associative memory and implicit processes: Theoretical and assessment issues. In: Wiers, R.; Stady, A., editors. Handbook of implicit cognition and addiction. Thousand Oaks, CA: Sage; 2006. p. 75-90.

Stacy A, Newcomb M, Bentler P. Cognitive motivation and drug use: A 9-year longitudinal study. Journal of Abnormal Psychology. 1991; 106:61-73. [PubMed: 9103718]

Sullivan EV, Pfefferbaum A. Neurocircuitry in alcoholism: A substrate of disruption and repair. Psychopharmacology. 2005; 180:583-594. [PubMed: 15834536]

Tiffany S. A cognitive model of drug urges and drug-use behavior: Role of automatic and nonautomatic processes. Psychological Review. 1990; 97:147-168. [PubMed: 2186423]

Townshend J, Duka T. Attentional bias associated with alcohol cues: Differences between heavy and occasional social drinkers. Psychopharmacology. 2001; 157:67-74. [PubMed: 11512045]

Waters H, Green W. A demonstration of attentional bias, using a novel dual task paradigm, toward clinically salient material in recovering alcohol abuse patients? Psychological Medicine. 2003; 33:491-498. [PubMed: 12701669]

Wiers, R.; Stacy, A., editors. Handbook of implicit cognition and addiction. Thousand Oaks, CA: Sage; 2006.

Wiers R, van Woerden N, Smulders F, de Jong P. Implicit and explicit alcohol-related cognitions in heavy and light drinkers. Journal of Abnormal Psychology. 2002; 111:648-658. [PubMed: 12428778] 